\title{
Tense Manages to Predict Implicative Behavior in Verbs
}

\author{
Ellie Pavlick \\ University of Pennsylvania \\ epavlick@seas.upenn.edu
}

\author{
Chris Callison-Burch \\ University of Pennsylvania \\ ccb@cis. upenn.edu
}

\begin{abstract}
Implicative verbs (e.g. manage) entail their complement clauses, while non-implicative verbs (e.g. want) do not. For example, while managing to solve the problem entails solving the problem, no such inference follows from wanting to solve the problem. Differentiating between implicative and non-implicative verbs is therefore an essential component of natural language understanding, relevant to applications such as textual entailment and summarization. We present a simple method for predicting implicativeness which exploits known constraints on the tense of implicative verbs and their complements. We show that this yields an effective, data-driven way of capturing this nuanced property in verbs.
\end{abstract}

\section{Overview}

Understanding language requires the ability to perform basic inference- to make conclusions about what is likely true or false based on what is said. For example, given the sentence She fixed the bug, we should almost certainly infer that the bug is fixed. However, rather than stating plainly that She fixed the bug, one might instead say:

(1a) She managed to fix the bug before midnight.

(1b) She happened to fix the bug while refactoring.

In either case, the hearer should still infer that the bug is fixed. But it is not as easy as always inferring that embedded clauses are true. By changing only one word, these sentence no longer give a clear indication as to whether or not the bug has been fixed: (2a) She wanted to fix the bug before midnight.

(2b) She planned to fix the bug while refactoring.

Implicative verbs, like those in (1), give rise to entailments, while non-implicative verbs, like those in (2), do not. It is therefore vital to natural language understanding to differentiate between clauses that are embedded under implicatives, which we can often infer to be either true or false, and those which are embedded under non-implicatives, for which such inferences cannot be made. In this paper, we exploit a known linguistic property of implicative verbs - that their complement clause is constrained to be in the same tense as the main clause- in order to predict the tendency of verbs to behave implicatively. We show that our method almost perfectly separates non-implicatives from implicatives in a small hand-labeled dataset, and that it provides strong signal for predicting entailments in sentences involving implicative verbs.

\section{Implicative Verbs}

Some English verbs can take infinitival complements, meaning they can appear in constructions of the form $V B_{1}^{*}$ to $V B_{2}$, where $V B_{1}^{*}$ is the "main" verb (which can be conjugated ${ }^{1}$ ) and $V B_{2}$ is the "complement" verb (which is in infinitive form). Examples (1a)-(2b) illustrate verbs taking infinitive complements.

Implicative verbs are a special subclass ${ }^{2}$ of such verbs which give rise to entailments involving their

\footnotetext{
${ }^{1}$ Here, * indicates that $\mathrm{VB}_{1}$ can match any verb form, e.g. $\mathrm{VB}, \mathrm{VBD}, \mathrm{VBP}$, etc. $\mathrm{VB}_{2}$ can only match the base form VB.

${ }^{2}$ We note that factive verbs represent another special class of verbs which can take infinitival complements. Unlike implica-
} 


\begin{tabular}{|lccl} 
& $\begin{array}{c}\text { Is the main verb } \\
\text { negated? }\end{array}$ & $\begin{array}{c}\text { Is the complement } \\
\text { entailed? }\end{array}$ & Example \\
Implicative & - & Yes & I managed to solve the problem. $\Rightarrow$ I solved the problem. \\
Implicative & + & No & I did not manage to solve the problem. $\Rightarrow$ I did not solve the problem. \\
Implicative & - & No & I failed to solve the problem. $\Rightarrow$ I did not solve the problem. \\
Implicative & + & Yes & I did not fail to solve the problem. $\Rightarrow$ I solved the problem. \\
Non-Impl. & - & Unknown & I wanted to solve the problem. $\neq$ I solved the problem. \\
Non-Impl. & + & Unknown & I did not want to solve the problem. $\not$ I did not solve the problem.
\end{tabular}

Table 1: Implicative verbs give rise to entailments involving their complement clauses. Non-implicatives entail neither the truth nor the falsity of their complements, and thus the truth/falsity of the complement is unaffected by negation of the main clause.

complement clauses. Individual implicatives can differ in the entailments they generate: e.g. while manage entails the truth of its complement, fail entails the falsity of its complement (failed to solve the problem $\Rightarrow$ didn't solve the problem). Despite these differences, however, implicatives represent a coherent class of verbs in that they permit some inference to be made about their complements, and this inference is sensitive to the context (positive/negated) of the main clause. This contrasts with non-implicative verbs, like want, which do not permit any inference regarding their complements, and for which the truth of the complement is unaffected by negation in the main clause (Table 1).

The method described in this paper aims to separate implicatives from non-implicatives (manage vs. want), rather than to differentiate between types implicatives (manage vs. fail). Making this implicative/non-implicative distinction is a necessary first step toward handling inferences involving embedded clauses, and one that, to date, has only been performed using manually-constructed word lists (MacCartney, 2009; Recasens et al., 2013).

\subsection{Tense Constraints on Complement Clauses}

Karttunen (1971) observed that, in sentences involving implicatives, the tense of the main verb must necessarily match the tense of the complement clause. For example, (3), in which the main clause and the complement are both in the past tense, is acceptable but (4), in which the complement is in the future, is clearly not. For non-implicatives, however,

tives, factives presuppose, rather than entail, their complements. E.g. both I was/was not glad to solve the problem entail I solved the problem. We do not address factives here, as factives rarely take infinitival complements: more often, they take "that" complements (e.g. know that, realize that). Factives that do take infinitival complements are mostly phrasal (e.g. be glad to). no such constraint exists: (6) is perfectly felicitous.

(3) I managed to solve the problem last night.

(4) \#I managed to solve the problem tomorrow.

(5) I planned to solve the problem last night.

(6) I planned to solve the problem tomorrow.

We exploit this property to predict implicativenesswhether the truth of a verb's complement can be inferred- by observing the verb's usage in practice.

\section{Method}

We hypothesize that, given a large corpus, we should be able to distinguish implicative verbs from nonimplicative verbs by observing how often the main verb tense agrees/disagrees with the tense of the complement clause. Unfortunately, verbs in infinitival complement clauses are not conjugated, and so are not necessarily marked for tense. We therefore use the Stanford Temporal Tagger (TT) (Chang and Manning, 2012) in order to identify time-referring expressions (e.g. tomorrow or last night) and resolve them to either past, present, or future tense.

We find all sentences containing $V B_{1}^{*}$ to $V B_{2}$ constructions in the Annotated Gigaword corpus (Napoles et al., 2012). We run the the TT over all of the sentences in order to identify time-referring expressions. We only consider sentences in which a time-referring expression appears and is in a direct dependency relationship with the complement verb $\left(V B_{2}\right)$. We provide the TT with the document publication dates, ${ }^{3}$ which are used to resolve each time mention to a specific calendar date and time. We then map these time expressions coarsely to either past, present, or future tense by comparing the

\footnotetext{
${ }^{3}$ Provided as metadata in the Annotated Gigaword.
} 
resolved time to the document creation time. Because of the fact that days were often not resolved correctly, or at all, we eventually throw away sentences in which the complement clause is labeled as present tense, as these are rarely true references to the present, and rather the result of incorrect time resolution, or implicit future references (e.g. I am going to solve the problem today implies the future as in later today, but this is not captured by the TT). We also assign the main clause to past, present, or future tense by using the fine-grained POS tag and a set of heuristics (for example, to check for modals). ${ }^{4}$

We assign a tense agreement score to each verb $v$ as follows. Let $S$ be the set of all $V B_{1}^{*}$ to $V B_{2}$ constructions in which $V B_{1}^{*}=v$. Then tense agreement is simply $\frac{1}{|S|} \times \mid\{s \in S \mid$ complement tense $=$ main tense $\} \mid$, i.e. the fraction of constructions in $S$ in which the tenses of the main and complement clauses agree. We expect implicative verbs to occur mostly in agreeing constructions, and thus have high tense agreement, while nonimplicatives may occur in both agreeing and nonagreeing constructions, and thus should have lower tense agreement. Note that while in theory, implicatives should never appear in non-agreeing constructions, the time annotation process is very imprecise, and thus we do not expect perfect results.

\section{Evaluation}

Recreating list from Karttunen (1971). Karttunen (1971) provides a short illustrative list of 7 known implicatives ${ }^{5}$ and 8 non-implicatives (shown in Table 2). As a first evaluation, we test whether tense agreement can accurately separate the verbs in this list, such that the implicatives are assigned higher agreement scores than the non-implicatives. Table 2 shows that this is indeed the case. Tense agreement almost perfectly divides the list, with implicative verbs appearing above non-implicative verbs in all cases. The one exception is decide (reportedly non-implicative), which appears above dare (reportedly implicative). This error, however,

\footnotetext{
${ }^{4}$ Full set of heuristics in supplementary material.

${ }^{5}$ The original list had 8 implicatives, but we omit remember since, in our data, it occurred almost exclusively with recurring time expressions, which we were not able to map to a specific date/time and thus tense, e.g. consumers must remember to make payments every 14 days.
}

seems understandable: while decide is not strictly implicative in the way manage is, it is often used as an implicative. E.g. the sentence I decided to leave would likely be taken to mean I left.

$\begin{array}{llll}\text { venture to } & 1.00 & \text { try to } & 0.42 \\ \text { forget to } & 0.80 & \text { agree to } & 0.34 \\ \text { manage to } & 0.79 & \text { promise to } & 0.22 \\ \text { bother to } & 0.61 & \text { want to } & 0.14 \\ \text { happen to } & 0.59 & \text { intend to } & 0.12 \\ \text { get to } & 0.52 & \text { plan to } & 0.10 \\ \text { decide to } & 0.45 & \text { hope to } & 0.03 \\ \text { dare to } & 0.44 & & \end{array}$

Table 2: Tense agreement scores for known implicatives (bold) and non-implicatives listed in Karttunen (1971). Ranking by tense agreement almost perfectly divides the two classes.

Predicting Entailment. Our interest is not in distinguishing implicatives from non-implicatives for its own sake, but rather to predict, based on the main verb, whether the truth of the complement can be inferred. We therefore conduct a second evaluation to assess how well tense agreement predicts this entailment property. We design our evaluation following the recognizing textual entailment (RTE) task (Dagan et al., 2006), in which two sentences are given, a premise $p$ and a hypothesis $h$, and the goal is to determine whether $p$ reasonably entails $h$. To construct our $p / h$ pairs, we take all the verbs extracted in Section 3 which appear in at least 50 tense-labeled sentences. For each of these verbs, we choose 3 random sentences in which the verb appears as $V B_{1}^{*}$ in a $V B_{1}^{*}$ to $V B_{2}$ construction. ${ }^{6}$ From each sentence, we extract the complement clause by deleting $V B_{1}^{*}$ to from the sentence, and conjugating $V B_{2}$ to match the tense of $V B_{1}^{*}$. We then use the original sentence as $p$ and the extracted complement as $h$ : e.g. a $p / h$ pair might look like I get to interact with fellow professors/I interact with fellow professors. We ask 5 independent annotators on Amazon Mechanical Turk to read each $p$ and then determine whether $h$ is true, false, or unclear given $p .^{7}$ We take the majority answer as the true label. We expect that implicative verbs should lead to judgements which are decidedly true or false while non-implicatives should lead

\footnotetext{
${ }^{6}$ These sentences can come from anywhere in the Gigaword corpus, they are not required to contain time expressions.

${ }^{7}$ Full annotation guidelines in supplementary material.
} 
(0.14) UFJ wants to merge with Mitsubishi, a combination that'd surpass Citigroup as the world's biggest bank. $\nRightarrow$ The merger of Japanese Banks creates the world's biggest bank.

(0.55) After graduating, Gallager chose to accept a full scholarship to play football for Temple University. $\Rightarrow$ Gallager attended Temple University.

(0.68) Wilkins was allowed to leave in 1987 to join French outfit Paris Saint-Germain. $\Rightarrow$ Wilkins departed Milan in 1987.

Table 3: Examples from the RTE3 dataset (Giampiccolo et al., 2007) which require recognizing implicative behavior, even in verbs that are not implicative by definition. The tendency of certain verbs (e.g. be allowed) to behave as de facto implicatives is captured surprisingly well by the tense agreement score (shown in parentheses).

to mostly judgements of unclear.

Figure 1 shows that these expectations hold. When a verb with low tense agreement appeared as the main verb of a sentence, the truth of the complement could only be inferred $30 \%$ of the time. When a verb with high tense agreement appeared as the main verb, the truth of the complement could be inferred $91 \%$ of the time. This difference is significant at $p<0.01$. That is, tense agreement provides a strong signal for identifying non-implicative verbs, and thus can help systems avoid false-positive entailment judgements, e.g. incorrectly inferring that wanting to merge $\Rightarrow$ merging (Table 3 ).

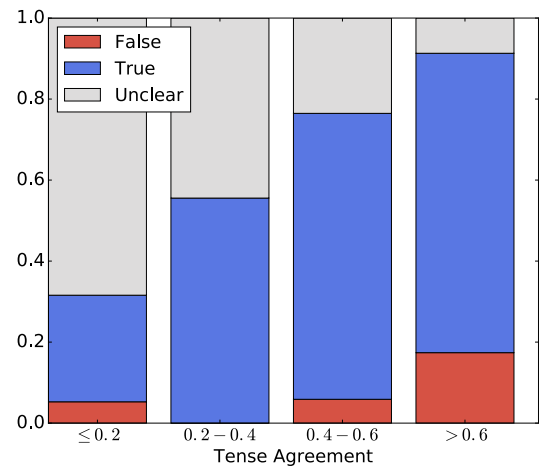

Figure 1: Whether or not complement is entailed for main verbs with varying levels of tense agreement. Verbs with high tense agreement yield more definitive judgments (true/false). Each bar represents aggregated judgements over approx. 20 verbs.

Interestingly, tense agreement accurately models verbs that are not implicative by definition, but which nonetheless tend to behave implicatively in practice. For example, our method finds high tense agreement for choose to and be allowed to, which are often used to communicate, albeit indirectly, that their complements did in fact happen. To convince ourselves that treating such verbs as implicatives makes sense in practice, we manually look through the RTE3 dataset (Giampiccolo et al., 2007) for examples containing high-scoring verbs according to our method. Table 3 shows some example inferences that hinge precisely on recognizing these types of $d e$ facto implicatives.

\section{Discussion and Related Work}

Language understanding tasks such as RTE (Clark et al., 2007; MacCartney, 2009) and bias detection (Recasens et al., 2013) have been shown to require knowledge of implicative verbs, but such knowledge has previously come from manually-built word lists rather than from data. Nairn et al. (2006) and Martin et al. (2009) describe automatic systems to handle implicatives, but require hand-crafted rules for each unique verb that is handled. The tense agreement method we present offers a starting point for acquiring such rules from data, and is well-suited for incorporating into statistical systems. The clear next step is to explore similar data-driven means for learning the specific behaviors of individual implicative verbs, which has been well-studied from a theoretical perspective (Karttunen, 1971; Nairn et al., 2006; Amaral et al., 2012; Karttunen, 2012). Another interesting extension concerns the role of tense in word representations. While currently, tense is rarely built directly into distributional representations of words (Mikolov et al., 2013; Pennington et al., 2014), our results suggest it may offer important insights into the semantics of individual words. We leave this question as a direction for future work.

\section{Conclusion}

Differentiating between implicative and nonimplicative verbs is important for discriminating inferences that can and cannot be made in natural language. We have presented a data-driven method 
that captures the implicative tendencies of verbs by exploiting the tense relationship between the verb and its complement clauses. This method effectively separates known implicatives from known non-implicatives, and, more importantly, provides good predictive signal in an entailment recognition task.

\section{Acknowledgments}

We would like to thank Florian Schwartz for valuable discussions. This research was supported by a Facebook Fellowship, and by gifts from the Alfred P. Sloan Foundation, Google, and Facebook. This material is based in part on research sponsored by the NSF grant under IIS-1249516 and DARPA under number FA8750-13-2-0017 (the DEFT program). The U.S. Government is authorized to reproduce and distribute reprints for Governmental purposes. The views and conclusions contained in this publication are those of the authors and should not be interpreted as representing official policies or endorsements of DARPA and the U.S. Government.

\section{References}

Patricia Amaral, Valeria de Paiva, Cleo Condoravdi, and Annie Zaenen. 2012. Where's the meeting that was cancelled? existential implications of transitive verbs. In Proceedings of the 3rd Workshop on Cognitive Aspects of the Lexicon, pages 183-194, Mumbai, India, December. The COLING 2012 Organizing Committee.

Angel X Chang and Christopher D Manning. 2012. Sutime: A library for recognizing and normalizing time expressions. In LREC, pages 3735-3740.

Peter Clark, Phil Harrison, John Thompson, William Murray, Jerry Hobbs, and Christiane Fellbaum. 2007. On the role of lexical and world knowledge in rte3. In Proceedings of the ACL-PASCAL Workshop on Textual Entailment and Paraphrasing, pages 54-59, Prague, June. Association for Computational Linguistics.

Ido Dagan, Oren Glickman, and Bernardo Magnini. 2006. The PASCAL recognizing textual entailment challenge. In Machine Learning Challenges. Evaluating Predictive Uncertainty, Visual Object Classification, and Recognising Tectual Entailment, pages 177190. Springer.

Danilo Giampiccolo, Bernardo Magnini, Ido Dagan, and Bill Dolan. 2007. The third PASCAL recognising textual entailment challenge. In Proceedings of the ACL-
PASCAL workshop on textual entailment and paraphrasing, pages 1-9.

Lauri Karttunen. 1971. Implicative verbs. Language, pages 340-358.

Lauri Karttunen. 2012. Simple and phrasal implicatives. In *SEM 2012: The First Joint Conference on Lexical and Computational Semantics - Volume 1: Proceedings of the main conference and the shared task, and Volume 2: Proceedings of the Sixth International Workshop on Semantic Evaluation (SemEval 2012), pages 124-131, Montréal, Canada, 7-8 June. Association for Computational Linguistics.

Bill MacCartney. 2009. Natural language inference. $\mathrm{Ph}$.D. thesis, Citeseer.

Fabienne Martin, Dennis Spohr, and Achim Stein. 2009. Disambiguation of polysemous verbs for rule-based inferencing. In Proceedings of the Eight International Conference on Computational Semantics, pages 222234, Tilburg, The Netherlands, January. Association for Computational Linguistics.

Tomas Mikolov, Kai Chen, Greg Corrado, and Jeffrey Dean. 2013. Efficient estimation of word representations in vector space. arXiv preprint arXiv:1301.3781.

Rowan Nairn, Cleo Condoravdi, and Lauri Karttunen. 2006. Computing relative polarity for textual inference. Inference in Computational Semantics (ICoS-5), pages 20-21.

Courtney Napoles, Matthew Gormley, and Benjamin Van Durme. 2012. Annotated gigaword. In Proceedings of the Joint Workshop on Automatic Knowledge Base Construction and Web-scale Knowledge Extraction (AKBC-WEKEX), pages 95-100, Montréal, Canada, June. Association for Computational Linguistics.

Jeffrey Pennington, Richard Socher, and Christopher D. Manning. 2014. Glove: Global vectors for word representation. In Empirical Methods in Natural Language Processing (EMNLP), pages 1532-1543.

Marta Recasens, Cristian Danescu-Niculescu-Mizil, and Dan Jurafsky. 2013. Linguistic models for analyzing and detecting biased language. In Proceedings of the 51st Annual Meeting of the Association for Computational Linguistics (Volume 1: Long Papers), pages 1650-1659, Sofia, Bulgaria, August. Association for Computational Linguistics. 